\title{
Proactive Coping and Professional Burnout in Special Education
}

\author{
Senada Duli \\ Ph.D Candidate, Faculty of Social Sciences, University of Tirana; nada_duli@hotmail.com
}

\section{Doi:10.5901/ajis.2015.v4n3s1p18}

\section{Abstract}

Proffessional burnout is a syndrom associated with a group symptoms such as emotional exhaustion, depersonalization and personal acomplishements. Proactive coping is considered those cognitive and behavioral efforts made to master and tolerate or reduce internal and external demands and conflicts among them. Special education teachers are in a direct contact with special needs of the students. They are more prone to experience burnout due to the way the perceive job demands and lack of personal resources to use coping strategies. The aim of this is to investigate correlations between burnout and proactive coping categories and to predict which of these correlation variables best predict emotional exhaustion as the main symptom of burnout. Results showed significant correlations between emotional exhaustion and strategic planning, preventing coping and emotional support seeking. Multiple regression analysis was used to predict which variable of proactive coping categories best predict emotional exhaustion. Emotional support seeking had statistic significance to predict emotional exhaustion for the total variances explained in this study.

Keywords: burnout, proactive coping, emotional exhaustion, emotional support seeking.

\section{Introduction}

The special education teachers have directly contact with students. They are responsible to meet their educational needs, like designing and implementing the Individualised Education Plan, evaluation and reevaluation, behavioral assessments, and other intervention plans. Students have different disabilities and the special education teacher has the responsibility to assist in every subject that these students meet some of the objectives as the same proficiency level as their general education peers. Special education teachers help general education teachers about accommodation of disabled students, about the strategies and methods they should use as they work with special needs students. The special education teacher contacts with a variety of individuals to facilitate the educational and general wellbeing of special needs students (Williams, Joy, Dikes, Catherine, 2015). Proffessional burnout is a syndrom associated with a group symptoms such as emotional exhaustion, depersonalization and personal acomplishements. The main definition of emotional exhaustion as the main symptom of burnout according to Maslach is the reduced energy level or extreme fatigue. Acording to Williams, Joy, Dikes, Catherine, 2015, job stress begins with emotional exhaustion which is the first reaction accompanied life events changes. Depersonalization is associated with negative emotions toward students or collegues. They may feel guilty regarding their work performance. Reduced self efficacy, is defined as negative self evaluation or minimizing personal acomplishements. Its a self evaluation process, that people use during their work. Maslach states that teacher working in special education services perceive low levels of job satisfaction and negative evaluation of job outcomes. Acording to Williams, Joy, Dikes \& Catherine, 2015), people experiencing burnout are more cynic, stressed and see the worst in everything. Various studies indicate, teachers working with students with special needs are more prone to experience job burnout. Most teachers of special education experience high levels of depression, psychological dysfunction and role conflict. One of the most important factors of professional burnout is direct contacts with students with special needs and perceieved lack of job success. Special education teachers have to complete several additional duties and responsabilites comparing to those of mainstream schools. (Zarafshan, Hadi, Mohammadi, Reza. M, Ahmadi, Fatemeh, Arsalani, Akram, 201. According to Maslach effects of burnout are widely experienced outside of work settings as well. A burnt out person may manifest these negative feeling toward his/her family members. (Williams, Joy, Dikes, Catherine, Education). There are many factors influencing teacher burnout. Lack of principals support, overload at work , lack of support resources and too many responsabilities at one time are the major factors increasing the possibility to put the special education teachers at risk of burnout. Teacher experience burnout when the their perceived stress overcomes their resources to cope with in an adequate way leading to exhaustion, cynism and low of personal acomplishements. Children of exhausted teachers struggle socially and emotionally, so they they achieve less goals in 
individualized education plan, giving a negative impact on their school performance. (Brunsting, et al., 2014). According to Plana, A., Fabregat,A., \& Gassio, J. (2003), coping is defined as those cognitive and behavioral efforts, developed for managing external and internal demands judged as exceeding or superpassing the individual own's resources. They define proactive coping as those cognitive and behavioral efforts made to master and tolerate or reduce internal and external demands and conflicts among them. Some people accumulate and use resources to prepare themselves for obstacles, so stressors are seen as challenges and they are more motivated to succeed. (Mackoniené, N. Norvilè, 2012). Billing and Moss identified three methods of coping strategies a) active-cognitive assessing potentially stressful events, b) active behavioral, efforts to manage a stressful situation c) avoidance, refuse to face the stressful situation. There are significant correlations between avoidance type of coping with burnout. The greater the feelings of emotional exhaustion, the more likely these professionals were to use strategies based on behavioral and mental disconnection from the situation when facing with a difficult or stressful event. According to Gil-Monte, Peiró and Valcárcel (1995) avoidance coping increased emotional exhaustion, while control coping maintain personal acomplishements at work. Is special education services, according to Wisniewski and Gargiulo (1997), shows that stress can not be avoided, but it can be managed through social support from administrative management, colleagues and superiors. (Plana,A., Fabregat, A., \& Gassio, J. 2003). Stressful situations create a discrapancy between desired state and perceived state. In this case coping may be activated in a direct and indirect way. In direct way stress activate coping directly before wellbeing has been threatened. Indirectly coping is activated after the individual wellbeing has been damaged. So, different coping strategies come before the process of burnout syndrome. Coping strategies oriented to the problem in combination with coping strategies focused to avoidance is more successful for for adaptation and wellbeing of the individuals. This is called according to as flexible coping with is more effective in controlling of work stressors. According to Schwarzer and Taubert (2002), they suggest a new conceptualization of coping, proactive, goal oriented and adaptive. Some people are more oriented to live their lives in such a way they accumulate resources and prepare themselves before stressful situations occur. They consider stressors as challenges and they are motivated to succeed and overpass them. (Plana,A., Fabregat, A., \&Gassio, J. 2003). Often stress is seen as a response as a adverse situation. They say that they feels a lot of stress. According to Schwarzer (2002), Selye distinguished a stressor (stimulus) from stress ( response). This response follows three stages: alarm reaction wich is a fight or flight response, is an adaptive short term reaction to emergency situations. Resistance stage, is the adaptation of the body to the stressor, the organism do not functional all well. The immune system is weak and many deseases have possibility to develop. In the exhaustion stage the organism has finished the resources. It activates the parasympathetic system, which may lead to burnout, depression and other different illness or even death. (Schwarzer, R., \& Taubert, S. 2002).

The stimulus based perspective aims to pay attention to the stressor causing a specific response. According to different researches adaptive efforts are necessary to cope with different situations. Characteristics of the stressors sometimes define the way how people react to that situation.

According to cognitive transactional paradigm it is important the cognitive appraisal of demands and the way of response. Our personal resources, like the social interactions with other especially in work settings, competencies, difficult situations are called antecedents according to Lazarus (2002), Schwarzer, R., \& Taubert, S. (2002) proactive people do not consider situations as harmful, loss or threat. They consider risks or demands as resources to help them toward promotion and personal growth. They see challengies not demands, they see goals attainement not risks. Due to this they increase their quality of functioning. Proactive coping improves life quality and people experience positive stress, wich leads to productivity and wellbeing. Different studies have been conducted in Germany, Poland and Canada to examine the relationship between proactive coping and job burnout. These studies show that proactive coping correlates negatively with job burnout in different professions. According to these studies proactive teachers perceive stress as challenging and less thraeatening than their reactive counterparts. (Schwarzer, R., \& Taubert, S. 2002).

In special education services teachers face different difficulties. This gives them greater possibility to leave their jobs. Sometimes children including ins special education system, are more stressed and experience more emotional problems due to the way how the teachers perceive their role. ( Major, E. A. 2012). Traditionally the focus of psychology has been on negative states than on positive one. Burnout represents a negative psychological state. Researchers attention now is focused on positive psychology based on well function. From this point of view burnout is supplemented by its positive antithesis of job engagement (Schaufeli, W.B., Leiter, M.P \& Maslach, Ch., 2001). Maslach and Leiter (2001), considered burnout as an engagement. According to them burnout starts with the wearing out of the engagement when energy turns into exhaustion, involvement turns into cynicism and efficacy turns into ineffectiveness. Engagement is assessed by the opposite pattern of scores of the three subscales of MBI. Unfavorable scores indicate burnout and favorable scores indicate engagement. (Schaufeli ,W.B., Leiter, M.P \& Maslach, Ch., 2008). If engagement is the 
opposite of burnout, then engagement profile is a match of sustainable workload, choice and control, recognition and reward, supportive work community, fairness and justice and meaningful work. According to Schaufeli (2008) engagement, is the positive anthithesis of burnout and it is assessed by two dimensions: activation and pleasure. So engagement is characterized by high levels of activation and pleasure. (Schaufeli,W.B., Leiter, M.P \& Maslach, Ch., 2001).

\section{The Pupose of this Study}

This is a pilot study which anticipate the final phase of gathering data. The pupose of this study is to show levels of experienced burnout among special education professionals and to examine significant correlations between job burnout subscales and proactive coping subscales in a group of 60 special education professionals. Also this study results aims to report which are the variables that best predict emotional exhaustion.

Research question 1:

Which are the levels of emotional exhaustion, depersonalization and personal acomplishements for special education professionals participating in this study?

Research question 3:

Are there significant correlations between job burnout subscales and proactive coping subscales?

Research question 2:

Which of the proactive coping categories best predicts emotional exhaustion?

\subsection{Research Methods}

\subsubsection{Sample}

This study was carried out with special education professionals in Tirana. A total number of 60 teachers, psychologiest and development therapiest were included in this study, only females. The way of cluster selection is with convenience. This is the pilot phase of the final study. The final phase will include a bigger sample, and will be extended in other cities of Albania where are situated special education schools, in urban environment. Subjects variate from 5 up to 30 years of works experience.

\subsubsection{Instruments}

\subsubsection{Maslach Burnout Inventory}

The items for the Maslach Burnout Inventory were designed to measure hypothesized aspects of the burnout syndrome. The scale is labeled at each point and ranges from 1 ( a few times a year or less), to 6 ( every day). A zero value is given if th respondent has never experienced the feeling or the attitude described (Maslach, Ch \& Jackson, S. E, 1981). Items are divided in three subscales; emotional exhaustion, depersonalization and personal acomplishements.

Reliability analysis coefficients are calculated for a sample of 20 subjects. Internal consistency is evaluated by Cronbach Coefficient's alpha. The realibility coefficients for the subscales were: for emotional exhaustion 0.89 , for depersonalization 0.90 and for personal acomplishements is 0.85 .

\subsubsection{Proactive Coping Inventory}

The Proactive Coping Inventory consists in seven scales and 55 items; one scale with 14 items measures exclusively proactive coping; the other sic scales focoses on positive facets of coping that strengthen coping inçitiative. The other six subscales are reflective coping, strategic planning, preventing coping, instrumental support seeking, emotional support seeking and avoidance coping. Answer scoring ranges from 1 (not at all true), 2 (barely true), 3 (somewhat true), to 4 (completely true). Three items of the proactive coping subscale have reverse scoring, "I like challengies and beating the odds", "I turn obstacles into positive experiences", and "When I have a problem, I usually see my self in a no win situation" (Greenglass, E., Schwarzer, R., Jakubiec, D., Fiksembaum, L \&Taubert, S, 1999). It was used alpha Cronbach coefficient for the reliability analysis. The reliability coefficients for Proactive Coping subscales in total is: 0.7 . 


\section{Analysis Results}

Results analysis of this quantitative research was conducted by using SPSS version 20. Descriptive analysis was used to calculate frequencies, means and standart deviation. The strength and the direction of the correlations between variables was determined using Pearson correlation.

The sample consists for 60 special education teachers, females. Ages ranked from 26 to 42 . Subjects years of works experience in that position ranked between 5 to 30 years of work.

The mean scores of the exhaustion is 15. This result show a low level of burnout. Depersonalization has a mean score of 2.9. Subjects report low levels of personalization.

Personal acomplishements have a mean score of 40. They report high levels of personal acomplishements, being included in the low category of burnout.

Results show a mean score of 47 for proactive coping subscale.

In reflective coping subscale the mean is 36. In strategic palnning respondents have a mean score of 13.9 . Preventing coping subscale has a mean score of 30 . Results show in instrumental seeking subscales a mean score of 24. 12 is the mean score for emotional seeking subscale. And 6 is the mean score for avoidance coping subscale.

\subsection{Correlations among variables}

\section{Table 1}

\begin{tabular}{|c|c|c|c|c|c|c|c|c|c|c|}
\hline & & 1 & 2 & 3 & 4 & 5 & 6 & 7 & 8 & 9 \\
\hline 1. & Personal acomplishements & 1 & & & & & & & & \\
\hline 2. & Depersonalization & $-.985^{\text {t* }}$ & 1 & & & & & & & \\
\hline 3. & Emotional exhaustion & $-.897^{\text {*t }}$ & $.917^{\text {*t }}$ & 1 & & & & & & \\
\hline 4. & Proactive coping & .298 & -.287 & -.143 & 1 & & & & & \\
\hline 5. & Reflective coping & .094 & -.159 & -.048 & $.485^{*}$ & 1 & & & & \\
\hline 6. & Strategic planning & .445 & $-.529^{*}$ & $-.553^{*}$ & .375 & $.567^{\star *}$ & 1 & & & \\
\hline 7. & Preventing planning & $.656^{* *}$ & $-.683^{* *}$ & $-.654^{* *}$ & .302 & $.609^{* *}$ & $.640^{\star *}$ & 1 & & \\
\hline 8. & Instrumental support & -.179 & .155 & .297 & $.514^{*}$ & $.542^{*}$ & .439 & -.043 & 1 & \\
\hline 9. & Emotional support & $.628^{* *}$ & $-.624^{\text {t* }}$ & $-.696^{* t}$ & .011 & .164 & .265 & $.464^{*}$ & -.215 & 1 \\
\hline 10. & Avoidance coping & -.040 & .173 & .058 & $-.476^{*}$ & $-.728^{* *}$ & $-.449^{*}$ & -.278 & $-631^{* *}$ & -.121 \\
\hline
\end{tabular}

**. Correlation is significant at the 0.01 level (2-tailed)

*. Correlation is significant at the 0.05 level (2-tailed).

There is a negative correlation between emotional exhaustion and strategic planning, preventing planning and emotional support. Subjects reported high levels of emotional exhaustion, scored low in strategic planning, preventing planning and emotional support.

Depersonalization also correlates negatively with strategic planning, preventing coping, and emotional support. There is a negative correlation between depersonalization and proactive coping in general. High scores of depersonalization received low scores in variables of strategic planning, preventing planning and emotional support.

Personal acomplishements correlates positively with preventing coping and emotional support seeking. Subjects which scored high in personal acomplishements and also received high scores in preventing coping and emotional support seeking.

\subsection{Prediction of the variables}

Table 2

\begin{tabular}{|lccccc|}
\hline Predictor variables & Beta & $\mathbf{t}$ & $\mathbf{p}$ & ${\text { Adj } \mathbf{R}^{2}}$ & Total variance \\
\hline Emotional exhaustion & & & & & \\
1.Strategic planning & -.255 & -1.214 & .246 & .584 & $58.4 \%$ \\
2.Preventing coping & -.253 & -1.109 & .288 & .584 & $58.4 \%$ \\
3.Emotional support & -.511 & -2.801 & .015 & .584 & $58.4 \%$ \\
\hline
\end{tabular}


It was used multiple linear regression analysis to predict wich of the variables explain better emotional exhausion (method enter was used). Emotional exhaustion is correlated with strategic planning, preventing coping and emotional support seeking. The three of these variables make contribution on predicting emotional exhaustion, Adj. $\mathrm{R}^{2}=.584 .58 \%$ of the total variance is explained by these three variables. This is statistically significant, $p<0.05$. But only emotional support seeking is statistically significant at $p<0.05$ level.

Results showed for strategic planning $\beta=-.255, t=-1.214, p>0.05$ for a total variance $19.6 \%$ Preventing coping $\beta=-.253, t=-1.109, p>0.05$, for a total variance of $17.9 \%$, for emotional support seeking, $\beta=-.511, t=-2.801, p<0.05$ for $a$ total variance $45.2 \%$. Higher levels of emotional exhaustion were linked to emotional support seeking. The best predictor for emotional exhaustion is emotional support seeking. This prediction is statistically significant at $p<0.05$ level.

\section{Discussion}

Results of this pilot study reveals low levels of exhaustion, low levels of depersonalisation and high levels of personal acomplishements. This was the first aim of this pilot study, whether there are low, moderate or high levels of burnout despite of the small sample.

The second aim of the study was to investigate the correlations between the burnout subscales and proactive coping subscales. Emotional exhaustion had a significant negative correlation with strategic planning, preventing coping and emotional support. Professionals scoring high in strategic planning, that use preventing coping before stressful events occurs at their work and they are emotionally supported are less prone to experience emotional exhaustion. The same variables are meaningful for depersonalization. Receiving high scores in strategic planning, preventing coping and emotional support, makes them to not experience levels of depersonalization. Emotional support seeking and using preventing strategies to cope successfully in work settings correlated positively with high scores in personal acomplishements. Also there were found significant correlations between burnout categories and years of experience at work. It was found a strong positive correlation between years of experience and emotional exhaustion. Increasing years of experience at work subjects reported more emotional exhaustion. These correlations may be explained, by having more responsibility during years, acumulutating more stress, using less coping strategies during their work and experience feelings of tiredness facing every day for years almost the same problems and difficulties.

The third aim of this study was to investigate wich variable best predict emotional exhaustion, as the main construct identifying burnout among correlated variables. The results showed that there were only three variables predicting emotional exhaustion, strategic planning, preventing coping and emotional support seeking. Regression analysis showed an adjusted $r$ square .584 , that means $58.4 \%$ of the variance may be explained by the these three variables predicting emotional exhaustion. Emotional support seeking was the best predictor of emotional exhaustion because this prediction has a significance level, $p=.015, p<0.05, \beta-.511, t=-2.801$. Using less strategies for emotional support during their work predicts levels of emotional exhaustion for the subjects of the special education.

This study has various limitations. First, the small number of subjects included do not represent the all population of special education professionals. These results in this phase are not completely representative. This is one of the first efforts made in my country recently to investigate burnout in relation to coping strategies. This sample was chosen for the pilot phase of study and will be extended at the final phase. At that phase burnout will be investigated in correlation with other variables, considered important provided from the literature and previous studies. As is well explained in various studies burnout syndrome inventory is used only for research studies purposes, and it is not a clinical evaluation.

\section{Conclusion}

Results show low levels of emotional exhaustion and depersonalization and high levels of personal acomplishements for special education professionals included in this study.

There were important relationships between burnout subscales and proactive coping subscales.

There were found significant negative correlations among emotional exhaustion and depersonalisation with strategic planning, preventing coping and emotional support seeking. There were also significant positive correlations between years of work experience and emotional exhaustion. Strategic planning, preventing coping, and emotional suppoer seeking were good predictors for emotional exhaustion but results of the linear multiple regression analysis, showd that only emotional support seeking was a significant factor for predicting emotional exhaustion. 


\section{References}

Brunsting, N.C., Streckovic, M., \& Lane. K. L. (2014). Special Education Teacher Burnout: A Synthesis of Research from 1979 to 2013. Journal of Education \& treatment of children, 37.

Greenglass, E., Schwarzer, R., Jakubiec, D., Fiksembaum, L \& Taubert, S. 1999, The Proactive Coping Inventory (PCl): A Multidimensional Research Instrument. (Online), Avaliable: http://userpage.fu-berlin.de/health/poland.htm (July 12-14, 1999).

Lee, S. M., Cho, S. H., Kissinger, D., \& Ogle.N.T. (2011). A typology of burnout in professional counselors, Journal of counseling \& development, 88, 131-138.

Mackoniene, R., \& Norvile, N. (2012). Burnout, Job satisfaction, self-efficacy, and proactive coping among Lithuanian school psychologists, TILTAI, 3, 199-210.

Major, A.E (2012). Job Design for Special Education Teachers, Current issues in education, 15, 1-9.

Maslach, Ch. \& Jackson, S. E. (1981). The measurement of experienced burnout. Journal of Occupational Behaviour, 2, 99-113.

Maslach, Ch., Schaufeli, W. B, \& Leiter. M.P. (2008). Job burnout, Carrier development international, 14, 204-220.

Maslach, Ch., Schaufeli, W. B., \& Leiter. M.P. (2001). Job burnout, Annual review psychology, 52, 397-422.

Plana, A. B., Fabregat, A. A., \& Gassio, J.B. (2003). Burnout syndrome and coping strategies: A structural relations model, Psychology in Spain, 7, 46-55.

Schwarzer, R., \& Taubert, S. (2002). Tenacious goal pursuits and striving toward personal growth: Proactive coping. In E. Freudenberg (Ed.), Beyond coping: Meeting goals, visions and challenges, 19-35.

Williams, Joy, Dikes, \& Catherine. (2015). The Implications of Demographic Variables as Related to Burnout among a Sample of Special Education Teachers, The new England Publishing Company, 135, 337-345.

Zarafshan, Hadi, Mohammadi, Reza.A., Ahmadi, Fatemeh, Arsalani, \& Akram. (2013). Job Burnout among Iranian Elementary School Teachers of Students with Autism: A Comparative Study, Iranian Journal of Psychiatry, 8, 20-27. 